en las primeras 24 horas siguientes al AIT inicial puede ayudar a identificar a los pacientes que presentan un riesgo elevado y que podrían beneficiarse más de tratamientos preventivos más agresivos. Hasta el momento, los datos clínicos son insuficientes para identificar a los pacientes en mayor riesgo.

En conclusión, este estudio confirma que las tasas de ACV recurrentes tempranos debidos a AGA son mayores que las observadas en otros subtipos clínicos. Estos resultados respaldan la necesidad de realizar pruebas imagenológicas urgentes transcraneales y de la carótida para identificar a los pacientes que presentan mayor riesgo. Algunos factores de riesgo y síntomas clínicos están asociados con determinados subtipos etiológicos de los AIT, pero aún se requieren factores más robustos de predicción de los ACV recurrentes para identificar a los pacientes con mayor riesgo según los diferentes subtipos clínicos de los AIT. (Purroy F, Montaner J, Molina CA, Delgado P, Ribo M, Álvarez-Sabín J. Patterns and predictors of early risk of recurrence after transient ischemic attack with respect to etiologic subtypes. Stroke. 2007;38;3225-9).

\section{Asociación entre el sobrepeso en la infancia y el riesgo de cardiopatía coronaria en la adultez}

Datos recientes demuestran que el número de niños con sobrepeso aumenta constantemente y la edad de comienzo de este trastorno es cada vez menor. Por ejemplo, según los Centros para el Control y la Prevención de Enfermedades de los Estados Unidos de América, 19\% de los niños entre 6 y 11 años de edad de ese país tienen un índice de masa corporal (IMC) mayor del percentil 95 para su edad y sexo.

Los factores de riesgo de cardiopatía coronaria, hipertensión arterial, dislipidemia, trastornos de la tolerancia a la glucosa y alteraciones vasculares ya están presentes en los niños con sobrepeso. Como resultado de la aparición temprana de esos factores de riesgo, el exceso de peso durante la niñez puede incrementar la probabilidad de sufrir alguna enfermedad cardíaca en la adultez.

Debido al incremento en el número de niños con sobrepeso, es imprescindible comprender mejor cuán graves pueden ser las consecuencias del exceso de peso durante la infancia con respecto al riesgo de padecer cardiopatía coronaria en la adultez. Para dilucidar esta interrogante se realizó un estudio de cohorte basado en la población en el que participaron 280678 personas nacidas en Dinamarca a partir de 1930. Se tomaron los datos de los controles médicos obligatorios anuales de 276835 niños (98,6\% del total) nacidos entre 1930 y 1976 en Copenhagen.
Tal como se esperaba, los valores de IMC (y sus desviaciones estándar) aumentaron con la edad. Durante los 46 años de seguimiento de este estudio se registraron 10235 casos con cardiopatía coronaria en hombres y 4318 en mujeres. En total, el estudio comprendió 5063622 personas-año de seguimiento.

Se encontró que los valores elevados del IMC durante la infancia aumentaron el riesgo de sufrir cardiopatía coronaria durante la adultez. Cada unidad de incremento en la puntuación z del IMC entre las edades de 7 y 13 años en los niños y de 10 y 13 años en las niñas elevó significativamente el riesgo de sufrir cardiopatía coronaria en la adultez. Esa asociación se hizo más fuerte a medida que aumentaba la edad dentro de esos intervalos. Como cada vez son más los niños y las niñas que aumentan de peso en todo el mundo, estos resultados indican que más niños y niñas se encuentran en riesgo de sufrir cardiopatía coronaria en la adultez.

La asociación significativa entre el aumento de la puntuación z del IMC entre los 7 y los 13 años de edad en los niños se mantuvo tanto para la cardiopatía coronaria en general como para su desenlace fatal y no fatal. A medida que los niños crecían, la distribución del IMC se amplió y el incremento de peso necesario para aumentar en una unidad la puntuación $\mathrm{z}$ del IMC a los 13 años de edad era más del doble del observado a los 7 años. Además del hecho de que la talla corporal al final de la niñez se asemeja más a la talla corporal de los adultos, los incrementos en las puntuaciones $\mathrm{z}$ del IMC en las edades más cercanas a la adultez reflejan una mayor acumulación de grasa, en particular de grasa abdominal, lo que aumenta el riesgo de cardiopatía coronaria.

En esta gran serie - con miles de casos y suficiente poder estadístico para detectar cualquier posible asociación - se confirmó que el peso al nacer no influye en la asociación entre el IMC en la infancia y la cardiopatía coronaria en la adultez. Desde el punto de vista de la salud pública, estos resultados tienen una gran importancia práctica, ya que demuestran que se deben concentrar los esfuerzos en intervenciones dirigidas a modificar el IMC durante la niñez - un factor de riesgo modificable- y le restan importancia al peso al nacer como factor de riesgo de cardiopatía coronaria en la adultez.

Este estudio de cohorte se basó en una población muy grande, que abarcó a casi todos los escolares de Copenhagen nacidos entre 1930 y 1976. A diferencia de otras investigaciones similares, el seguimiento en este estudio fue completo y los datos estuvieron disponibles para la totalidad de los participantes. Teniendo en cuenta que solamente 3,1\% de la población de Copenhagen no era de origen europeo durante los años del estudio, se puede asu- 
mir que estos resultados se pueden generalizar a otras poblaciones de similar composición étnica.

La asociación lineal encontrada entre el IMC durante la niñez y la cardiopatía coronaria en la adultez indica que cada vez más niños enfrentan un mayor riesgo de sufrir cardiopatía coronaria cuando sean adultos. Se calculó que para un niño de 13 años de edad que pese $11,2 \mathrm{~kg}$ más que el promedio de niños de su edad, la probabilidad de sufrir cardiopatía coronaria antes de cumplir 60 años es $33 \%$ mayor que el de sus coetáneos sin sobrepeso. Sin embargo, como la magnitud del riesgo es moderada a los 7 años y aumenta considerablemente a la edad de 13 años, estos resultados indican que es posible y necesario realizar intervenciones en este período de la infancia para reducir el riesgo de cardiopatía coronaria una vez que lleguen a la edad adulta. (Baker JL, Olsen LW, Sørensen TIA. Childhood body-mass index and the risk of coronary heart disease in adulthood. 2007;357:2329-37). 